\title{
Knockdown of Ror2 suppresses TNF- $\alpha$-induced inflammation and apoptosis in vascular endothelial cells
}

\author{
XIAOHU YANG, SUMING ZHAO, HONGXIN YUAN, RONGFENG SHI, \\ WEIWEI GU, ZHUXIN GU, XIAOHUA LU and HUI ZHAO
}

Department of Intervention Radiology, Affiliated Hospital of Nantong University, Nantong, Jiangsu 226000, P.R. China

Received January 20, 2020; Accepted June 9, 2020

DOI: $10.3892 / \mathrm{mmr} .2020 .11377$

\begin{abstract}
Wnt family member 5a (Wnt5a) is a noncanonical member of the Wnt family that is highly expressed in atherosclerosis. Studies have shown that Wnt5a/receptor tyrosine kinase-like orphan receptors 2 (Ror2) signaling can participate in the formation of foam cells; however, the role of Ror2 in vascular endothelial cells during atherosclerotic injury is unknown. Therefore, the present study aimed to investigate the role of Ror2 in tumor necrosis factor (TNF)- $\alpha$-induced vascular endothelial cell injury and investigate whether it could be regulated by Wnt5a. Human umbilical vein endothelial cells were transfected with short hairpin RNA specific against Ror2 in the absence or presence of TNF- $\alpha$. The alteration of inflammatory cytokine levels was detected, and the expression of adhesion molecules was assessed. Western blot and flow cytometry analyses were used to detect the activation of nuclear factor- $\mathrm{\kappa B}(\mathrm{NF}-\kappa \mathrm{B})$ signaling and cell apoptosis. The interaction between Ror2 and Wnt5a was confirmed by immunoprecipitation. Ror2 was upregulated upon TNF- $\alpha$ stimulation. Knockdown of Ror2 inhibited the TNF- $\alpha$-induced release of inflammatory cytokines, the expression of intercellular adhesion molecule- 1 and vascular cell adhesion molecule- 1 and the activation of NF- $\kappa \mathrm{B}$ signaling. Furthermore, cell apoptosis induced by TNF- $\alpha$ was rescued by Ror2 silencing. In addition, Wnt5a expression was increased by TNF- $\alpha$, and Ror 2 could bind to Wnt5a, the knockdown of which could downregulate the levels of Ror2. In conclusion, it was demonstrated that Ror2 was upregulated upon TNF- $\alpha$ stimuli, and interference of Ror 2 regulated by Wnt5a could suppress TNF- $\alpha$-induced inflammation and apoptosis in vascular endothelial cells.
\end{abstract}

Correspondence to: Dr Hui Zhao, Department of Intervention Radiology, Affiliated Hospital of Nantong University, 20 Xisi Road, Nantong, Jiangsu 226000, P.R. China

E-mail: zhaohlab@163.com

Key words: atherosclerosis, receptor tyrosine kinase-like orphan receptors, tumor necrosis factor- $\alpha$, vascular system injuries, Wnt proteins

\section{Introduction}

Atherosclerosis (AS) is a gradual process of thickening and sclerosis of the vascular wall caused by lipid accumulation, fibrosis and calcium deposition in the intima of the affected artery, characterized by subintimal fibrous plaques and/or atheromas (1). Subsequently, AS can cause ischemic and hypoxic changes in the corresponding tissues and organs, thus leading to a series of complications, including coronary artery disease, hypertension, cerebral infarction and peripheral arterial disease (2). The specific pathogenesis of AS is complex and involves multiple pathophysiological processes, such as inflammation, endothelial cell injury, immunoreaction, autophagy and apoptosis $(3,4)$.

Vascular endothelial cell injury or endothelial dysfunction is involved in the initiation and progression of AS (2). Vascular endothelial cells are predominantly composed of flat epithelial cells on the inner surfaces of the heart, blood vessels and lymphatic vessels (5). Besides acting as a barrier between blood and blood vessel walls, vascular endothelial cells can synthesize and release a variety of endothelial-derived vasoactive factors, therefore exerting various physiological functions, such as reducing vascular permeability, inhibiting cell migration and chemotaxis, regulating vasoconstriction and relaxation, preventing platelet aggregation and anti-adhesion (5). Upon the stimulation of various inflammatory factors, the functions and structure of endothelial cells are damaged, triggering a series of inflammatory reactions that involved a large number of inflammatory mediators (6). Damaged vascular endothelial cells express adhesion molecules, such as intercellular adhesion molecule-1 (ICAM-1) and vascular cell adhesion molecule-1 (VCAM-1), which can bind to monocytes and lymphocytes in the blood and cause adhesion reactions (7). Subsequently, large amounts of chemical chemokines are produced and released, which promotes the accumulation, migration and deposition of monocytes and lymphocytes under the vascular endothelium. Monocyte chemoattractant protein 1 (MCP-1) and monocyte/ macrophage colony stimulating factor promotes the transformation of monocytes into macrophages, which can form foam cells by uptake of oxidized low density lipoprotein (8). A large number of inflammatory factors, such as interferon (IFN) $-\gamma$, tumor necrosis factor (TNF)- $\alpha$, and interleukin (IL)-1 are then released, which in turn further stimulates and exacerbates the 
inflammatory response of lymphocytes, macrophages, foam cells and endothelial cells (9). In the present study, TNF- $\alpha$ was used to induce vascular inflammation that could contribute to AS in vitro.

Wnts are a family of highly conserved, secreted glycoproteins involved in numerous biological processes, such as proliferation, differentiation, polarity, cell survival and adhesion (10). Wnts can activate at least two distinct signaling pathways; the canonical Wnt/ $\beta$-catenin and noncanonical pathways, where the latter can be further classified into the Wnt/ $\mathrm{Ca}^{2+}$ and the planar cell polarity (PCP) pathways (10). Over the past decades, the Wnt pathway was reported to play a key role in the occurrence and development of inflammatory diseases, including AS (11). During the development of AS, pro-inflammatory factors, such as TNF- $\alpha$, IFN- $\gamma$ and nitric oxide, can activate the Wnt pathway; the signal transduction of which can be maintained and enhanced by IL-6 and TNF- $\alpha$ (12). In addition, the Wnt pathway in turn has a pro-inflammatory effect, which can promote the release of inflammatory factors and concurrently contribute to endothelial dysfunction, resulting in plaque formation (12). Wnt family member 5a (Wnt5a), a member of the Wnt family, was first identified in the early 1990s, and was shown to play an important role in AS. Christman et al (13), demonstrated Wnt5a expression in human and murine atherosclerotic lesions for the first time. Since then, numerous studies have reported the elevated expression of Wnt5a in human atherosclerotic lesions and the serum of patients with AS $(14,15)$.

The receptor tyrosine kinase like orphan receptor 2 (Ror2) is a member of the Ror family that is essential for cell migration, skeletal and nervous system development and oncogenesis (16). Ror2 acts as a receptor or co-receptor for Wnt5a that is critical for activation of the PCP signaling pathway by Wnt5a (17). The Wnt5a-Ror2 signaling pathway plays an important role in regulating tumor cell growth, directional migration, invasion and cell polarity during organogenesis (18). The Wnt5a-Ror2 axis has been demonstrated to be involved in inflammation (19,20). Ror2 and Wnt5a expression levels have been found to be elevated in atherosclerotic lesions and participate in the formation of atherosclerotic foam cells (21). However, the functions of Ror2 in vascular endothelial cell injury of AS remain to be clarified.

Taken together, the present study aimed to investigate whether Ror 2 could protect vascular endothelial cells against TNF- $\alpha$-induced inflammation and apoptosis. Additionally, the modulatory effect of Wnt5a on Ror2 was explored.

\section{Materials and methods}

Cell culture and treatment. Human umbilical vein endothelial cells (HUVECs) were purchased from American Type Culture Collection and maintained in RPMI-1640 medium (Thermo Fisher Scientific, Inc.) supplemented with $10 \%$ heat-inactivated FBS (Thermo Fisher Scientific, Inc.) and $100 \mathrm{U} / \mathrm{ml}$ penicillin G. Cells were incubated at $37^{\circ} \mathrm{C}$ in a humidified atmosphere containing $5 \% \mathrm{CO}_{2}$ and $95 \%$ air.

To induce inflammatory response in the HUVECs, cells were treated with different concentrations of TNF- $\alpha(0,2.5,5$, or $10 \mathrm{ng} / \mathrm{ml}$; PMC3016; Thermo Fisher Scientific, Inc.) at $37^{\circ} \mathrm{C}$ for $24 \mathrm{~h}(22)$.
Plasmids and transfection. Short hairpin RNA (shRNA) sequences targeting Ror2 (-1: 5'-CCGGCCGCTACCATCAGTGCTATAACTCGAGTTATAGCACTGATGGTAGC GGTTTTT-3'; -2: 5'-GCCCGAT TCCAACTCTGA AAG-3') and Wnt5a (-1: 5'-C-CGGCCTGTTCAGATGTCA GAAGTACTCGAGTACTTCTGACATCTGAACAGGTTT TTG-3'; -2: 5'-TGGTGCTGCTATGTCAAATGCAAG ATTCAAGAGA TCTTGCATTTGACATAGCAGCACC TTTTTTC-3') were designed and constructed, then cloned into plasmids (Santa Cruz Biotechnology, Inc.). A total $2 \mu \mathrm{g} / \mathrm{ml}$ of Ror2 and Wnt5a shRNA plasmids or scrambled shRNAs (5'-GCCCAGCCAAGACATGGAAAT-3') were transfected into HUVECs using Lipofectamine ${ }^{\mathrm{TM}} 2000$ reagent (Invitrogen; Thermo Fisher Scientific, Inc.). Cells were then transferred to normal RPMI-1640 culture medium containing $10 \%$ FBS and cultured for $48 \mathrm{~h}$. Ror 2 and Wnt5a expression levels were measured by reverse transcription-quantitative PCR (RT-qPCR) assays.

Western blotting and immunoprecipitation (IP) assays. Total proteins of HUVECs were extracted using RIPA lysis buffer (Thermo Fisher Scientific, Inc.) with Complete EDTA Free Protease and Phosphatase Inhibitors (Roche Diagnostics). Individual protein concentrations were determined using a bicinchoninic acid assay kit (Thermo Fisher Scientific, Inc.). A total of $10 \mu \mathrm{g}$ samples were then separated by $12 \%$ gradient sodium dodecyl sulfate polyacrylamide gel electrophoresis, transferred to polyvinylidene fluoride membranes (Thermo Fisher Scientific, Inc.), blocked with 5\% non-fat milk at room temperature for $2 \mathrm{~h}$, and incubated overnight with specific primary antibodies. The primary antibodies (all purchased from Santa Cruz Biotechnology, Inc.) used were as follows: Ror2 (cat. no. sc-374174; 1:500), ICAM-1 (cat. no. sc-8439; 1:500), VCAM-1 (cat. no. sc-13160; 1:500), phosphorylated (p)-ІкB $\alpha$ (cat. no. sc-52900; 1:200), p65 (cat. no. sc-8008; 1:500), ІкB $\alpha$ (cat. no. sc-1643; 1:400), lamin B (cat. no. sc-374015; 1:200), Bcl-2 (cat. no. sc-7382; 1:1,000), Bax (cat. no. sc-7480; 1:1,000), cleaved-caspase 3 (cat. no. sc-271759; 1:200), cleaved-caspase 7 (cat. no. sc-56067; 1:200), caspase 3 (cat. no. sc-7272; 1:500), caspase 7 (cat. no. sc-56063, 1:500), Wnt5a (cat. no. sc-365370; 1:500) and GAPDH (cat. no. sc-47724; 1:1,000). GAPDH was used as the loading control. Following overnight incubation at $4^{\circ} \mathrm{C}$, membranes were incubated with horseradish peroxidase-conjugated secondary antibodies (goat anti-rabbit, cat. no. sc-2004, 1:10,000 and goat anti-mouse, cat. no. sc-2005, 1:10,000; Santa Cruz Biotechnology, Inc.). Finally, membranes were visualized using the ECL system and ImageJ software (v1.46r; National Institutes of Health) was used to quantify the intensity of each protein band.

The interaction between Ror2 and Wnt5a proteins in HUVECs was validated using IP. For the IP assay, soluble protein samples were pre-incubated with protein G/A-agarose (Cell Signaling Biotechnology, Inc) at $4^{\circ} \mathrm{C}$ overnight and then incubated with $100 \mu \mathrm{l}$ protein G/A-agarose pre-coupled to antibody against primary antibodies for $\geq 3 \mathrm{~h}$ at room temperature. The mixtures were then washed with PBS, boiled and subjected to western blotting.

$R T$ - $q P C R$. Total RNA was isolated using TRIzol ${ }^{\circledR}$ reagent (Invitrogen; Thermo Fisher Scientific, Inc.) and reverse tran- 
A
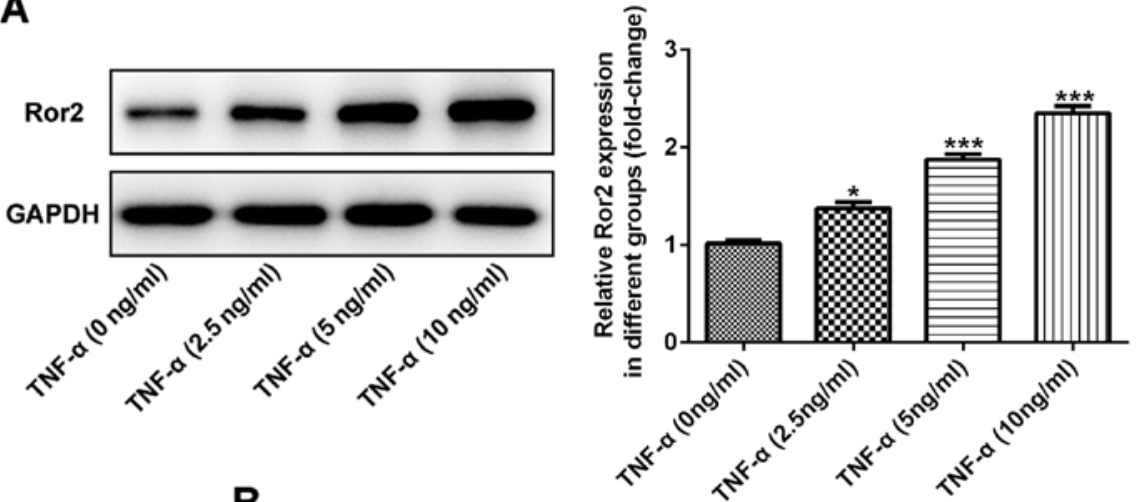

B

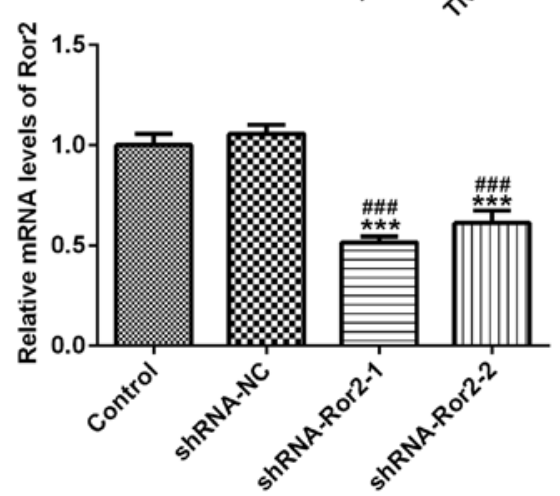

Figure 1. Ror2 is upregulated upon TNF- $\alpha$ stimulation in HUVECs. (A) Representative immunoblot analysis and relative protein expression of Ror2 in response to different concentrations of TNF- $\alpha . \mathrm{n}=3$. ${ }^{*} \mathrm{P}<0.05$ and ${ }^{* * *} \mathrm{P}<0.001$ vs. TNF- $\alpha(0 \mathrm{ng} / \mathrm{ml})$. (B) Relative mRNA expression of Ror2 in different groups. $\mathrm{n}=3$. ${ }^{* * *} \mathrm{P}<0.001$ vs. control; \#\#" $\mathrm{P}<0.001$ vs. shRNA-NC. TNF- $\alpha$, tumor necrosis factor- $\alpha$; shRNA, short hairpin RNA; NC, negative control; Ror2, receptor tyrosine kinase-like orphan receptors 2; HUVEC, human umbilical vein endothelial cells.

scribed to cDNA using the PrimeScript RT reagent kit with gDNA Eraser (Takara Bio, Inc.), according to the manufacturer's instructions. A total of 50 ng cDNA was subsequently used for qPCR using TB Green ${ }^{\circledR}$ Fast qPCR Mix (Takara Biotechnology Co., Ltd.). The following primers were used: Ror2 forward, 5'-TTACAGAGGAACGGCAAGCA-3' and reverse, 5'-CTGCTGTCTCGGGGACGTTT-3'; Wnt5a forward, 5'-ATTCTGGCTCCACTTGTTGCT-3' and reverse, 5'-TTCATACCTAGCGACCACCA-3'; ICAM-1 forward, 5'-ATGGCAACGACTCCTTCTCG-3' and reverse, 5'-GCCGGAAAGCTGTAGATGGT-3'; and VCAM-1 forward, 5'-TGGATAATGTTTGCAGCTTCTCA-3' and reverse, 5'-CGTCACCTTCCCATTCAGTG-3'. Human GAPDH was used as the control with the following sequences: Forward, 5'-AATGGGCAGCCGTTAGGAAA-3' and reverse, 5'-AATGGGCAGCCGTTAGGAAA-3'. The following thermocycling conditions were used for the qPCR: Initial denaturation at $95^{\circ} \mathrm{C}$ for $30 \mathrm{sec}$; and 40 cycles of $95^{\circ} \mathrm{C}$ for $5 \mathrm{sec}$ and $60^{\circ} \mathrm{C}$ for $15 \mathrm{sec}$, followed by default of melt curve (Applied Biosystems 7500; Thermo Fisher Scientific, Inc.). Differential expression of mRNA was calculated using the $2^{-\Delta \Delta C q}$ method (23).

Enzyme-linked immunosorbent assay (ELISA). The generation of inflammatory factors TNF- $\alpha$ (cat. no. ab181421), IL-1 $\beta$ (cat. no. ab100562), IL-6 (cat. no. ab178013) and MCP-1 (cat. no. ab179886) was determined using ELISA kits (Abcam) according to the manufacturer's instructions. Briefly, cell supernatants were added to 96-well plates, and then incubated with biotin-conjugated antibodies (included in the kits) targeting TNF- $\alpha$, IL-1 $\beta$, IL- 6 and MCP- 1 at $37^{\circ} \mathrm{C}$ for $1 \mathrm{~h}$.
Following incubation with working solution at $37^{\circ} \mathrm{C}$ for $30 \mathrm{~min}$ and TMB solution for 15 min in darkness, the absorbance at a wavelength of $450 \mathrm{~nm}$ was detected using a microplate reader (Thermo Fisher Scientific, Inc.).

Flow cytometry. Cell apoptosis was assessed by flow cytometry using propidium iodide (PI) staining. In brief, cells were gently washed twice with PBS, digested with $0.25 \%$ trypsin and centrifuged at $200 \mathrm{x}$ g for $5 \mathrm{~min}$ at $4^{\circ} \mathrm{C}$. Following resuspension of the cell pellet with $1 \mathrm{ml} \mathrm{NaCl} / \mathrm{Pi}$, cells were incubated with PI for $15 \mathrm{~min}$ in a dark room at room temperature and immediately analyzed using a flow cytometer (Becton, Dickinson and Company). Data were analyzed by flow cytometry software (iSort Automated Cell Sorter, vA.0; Thermo Fisher Scientific, Inc).

Statistical analysis. All experiments were repeated at least three times and data are expressed as the mean \pm standard deviation. Student's t-test was used for comparison between two groups, one-way ANOVA was used for comparison among multiple groups and Tukey's post hoc test was used for pairwise comparison. $\mathrm{P}<0.05$ was considered to indicate a statistically significant difference (24).

\section{Results}

Ror2 is upregulated by TNF- $\alpha$ stimuli in HUVECs. Firstly, the protein expression of Ror2 in HUVECs treated with different concentrations of TNF- $\alpha$ was detected by western blotting. As presented in Fig. 1A, Ror2 expression was enhanced by 

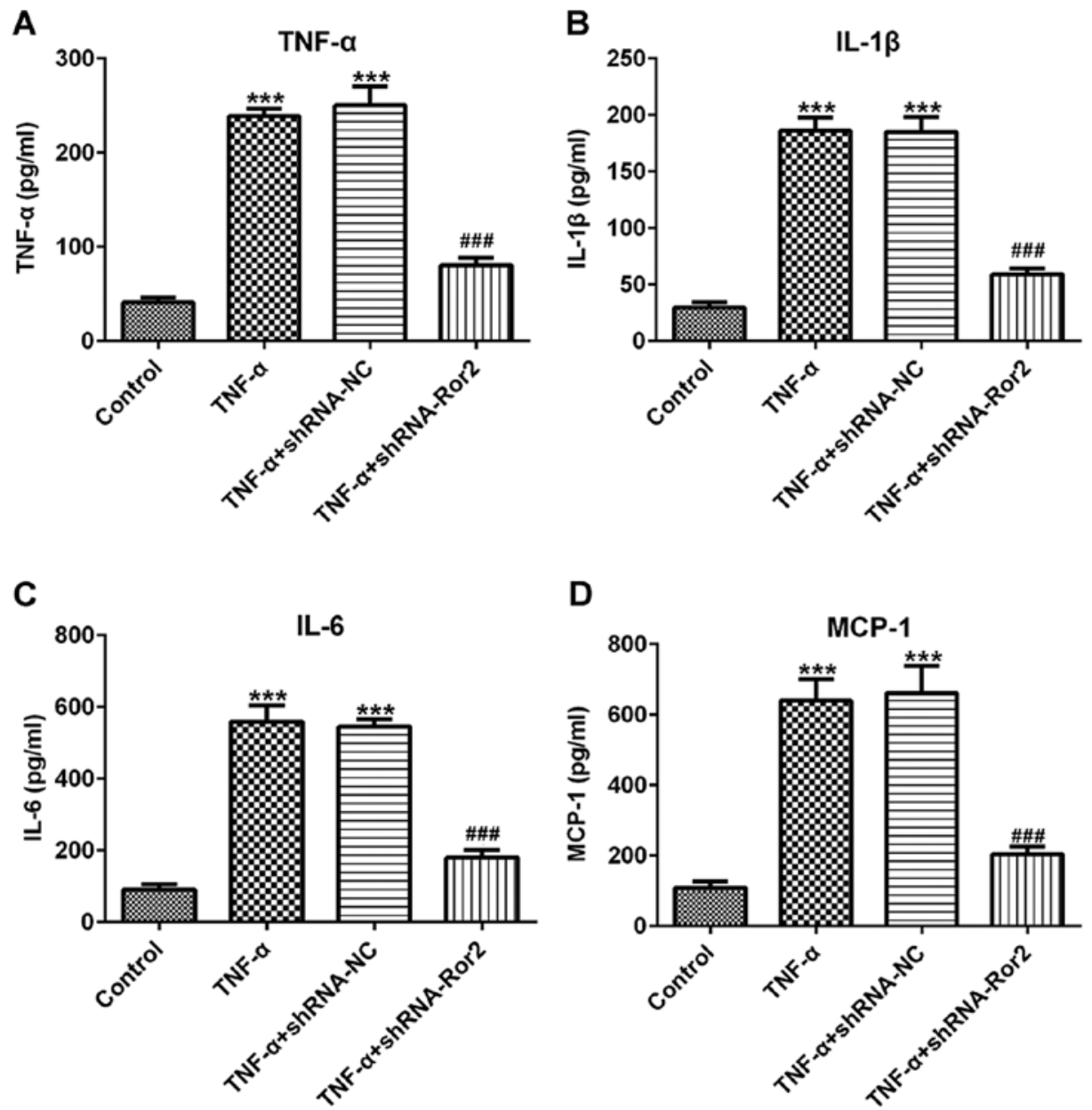

Figure 2. Ror2 knockdown inhibits the release of inflammatory cytokines. Cells transfected with or without shRNA-Ror2/shRNA-NC were exposed to $10 \mathrm{ng} / \mathrm{ml} \mathrm{TNF}-\alpha$ for $24 \mathrm{~h}$, and then cells were lysed to measure the contents of (A) TNF- $\alpha$, (B) IL-1 $\beta$, (C) IL-6 and (D) MCP-1 by ELISA. $\mathrm{n}=3$. ${ }^{* * *} \mathrm{P}<0.001 \mathrm{vs}$. control; ${ }^{\# \# ~} \mathrm{P}<0.001$ vs. TNF- $\alpha$. TNF- $\alpha$, tumor necrosis factor- $\alpha$; IL, interleukin; MCP, monocyte chemoattractant protein; shRNA, short hairpin RNA; NC, negative control; Ror2, receptor tyrosine kinase-like orphan receptors 2.

TNF- $\alpha$ in a concentration-dependent manner, suggesting the potential modulatory effect of Ror2 on TNF- $\alpha$ dose-dependent increase in vascular endothelial cell injury. This result was in accordance with a previous study, in which Ror2 expression was reported to be significantly expressed at a higher level in advanced human atherosclerotic lesions compared with less advanced lesions (21). Furthermore, the expression level of Ror 2 was the highest following treatment with $10 \mathrm{ng} / \mathrm{ml}$ TNF- $\alpha$. Therefore, $10 \mathrm{ng} / \mathrm{ml}$ was selected as the concentration of TNF- $\alpha$ to be used in the following experiments based on the present and previous results $(22,25,26)$. To further investigate the effects of Ror2 on TNF- $\alpha$-induced vascular endothelial cell injury, two shRNA expression vectors, shRNA-Ror2-1 and shRNA-Ror2-2, were used to knock down the expression of Ror2 in HUVECs, and cells transfected with empty plasmids were used as a negative control. The results revealed a more efficient knockdown effect of shRNA-Ror2-1 compared with shRNA-Ror2-2. shRNA-Ror2-1 produced a nearly 50\% reduction in the expression level of Ror2, which is significant and enough to affect downstream inflammatory response (27), therefore it was selected for subsequent experiments (Fig. 1B).

Knockdown of Ror2 inhibits TNF- $\alpha$-induced inflammation in HUVECs. It was then investigated whether Ror2 silencing could protect HUVECs against TNF- $\alpha$-induced injury. As presented in Fig. 2, TNF- $\alpha$ treatment resulted in a significant increase in the release of inflammatory cytokines, including TNF- $\alpha$, IL-1 $\beta$, IL- 6 and MCP-1 compared with controls. Meanwhile, cells with Ror2-knockdown released significantly fewer inflammatory cytokines compared with the TNF- $\alpha$ groups. Results from RT-qPCR and western blotting revealed that compared with controls, TNF- $\alpha$ resulted in a significant increase in the mRNA and protein expression levels of adhesion molecules ICAM-1 and VCAM-1, whereas Ror2-silencing significantly decreased the mRNA and protein expression of ICAM-1 and VCAM-1 (Fig. 3), indicating the inhibitory effect of Ror2-knockdown on TNF- $\alpha$-induced inflammation in endothelial cells.

Knockdown of Ror 2 obstructs the TNF- $\alpha$-induced activation of $N F-\kappa B$. NF- $\kappa \mathrm{B}$ is a protein complex consisting of p65 and p50 (28). Following binding to TNF- $\alpha$ receptor, TNF- $\alpha$ can activate IкB $\alpha$ kinase, which plays a role in phosphorylating

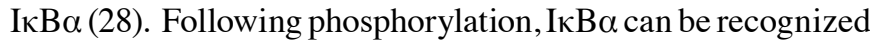
and degraded by the proteasomes (28). As a result, NF- $\kappa B$ is activated by release from the cytoplasmic NF- $\kappa \mathrm{B} / \mathrm{I} \kappa \mathrm{B} \alpha$ complex and translocation into the nucleus, thereby activating the expression of target genes, such as TNF- $\alpha$ and IL-1 (28). 
A

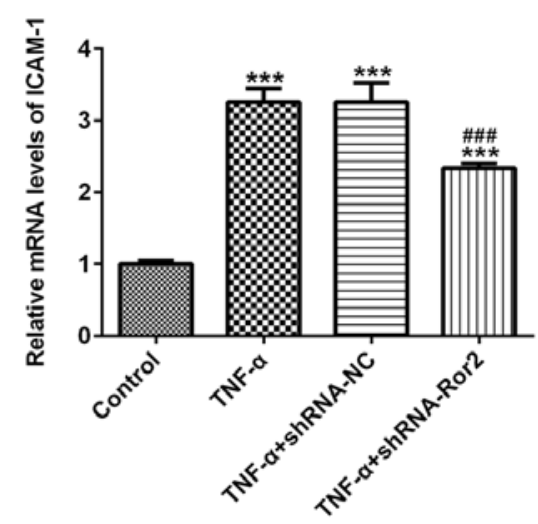

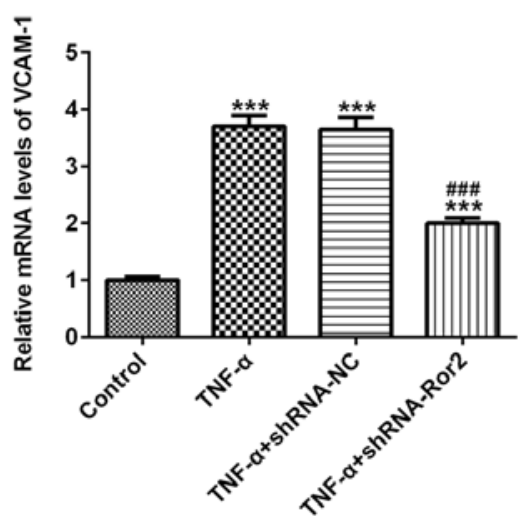

B
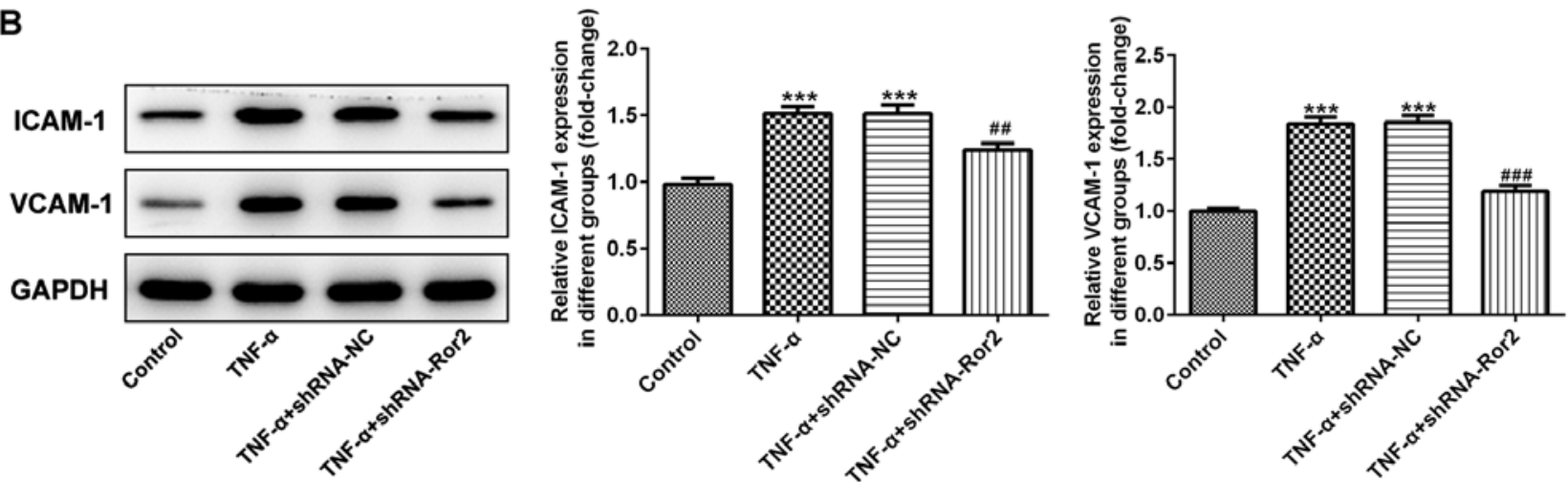

Figure 3. Ror2-knockdown inhibits the expression of ICAM-1 and VCAM-1. (A) Relative mRNA levels of ICAM-1 and VCAM-1 in different groups. $\mathrm{n}=3$. (B) Representative immunoblot analysis and relative protein expression of ICAM-1 and VCAM-1 in different groups. $\mathrm{n}=3$. ${ }^{* * * *} \mathrm{P}<0.001 \mathrm{vs}$. control; ${ }^{\# \#} \mathrm{P}<0.05$

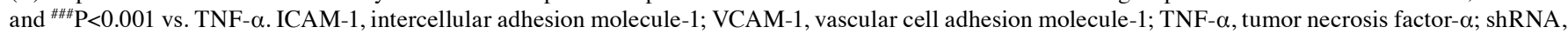
short hairpin RNA; NC, negative control; Ror2, receptor tyrosine kinase-like orphan receptors 2.
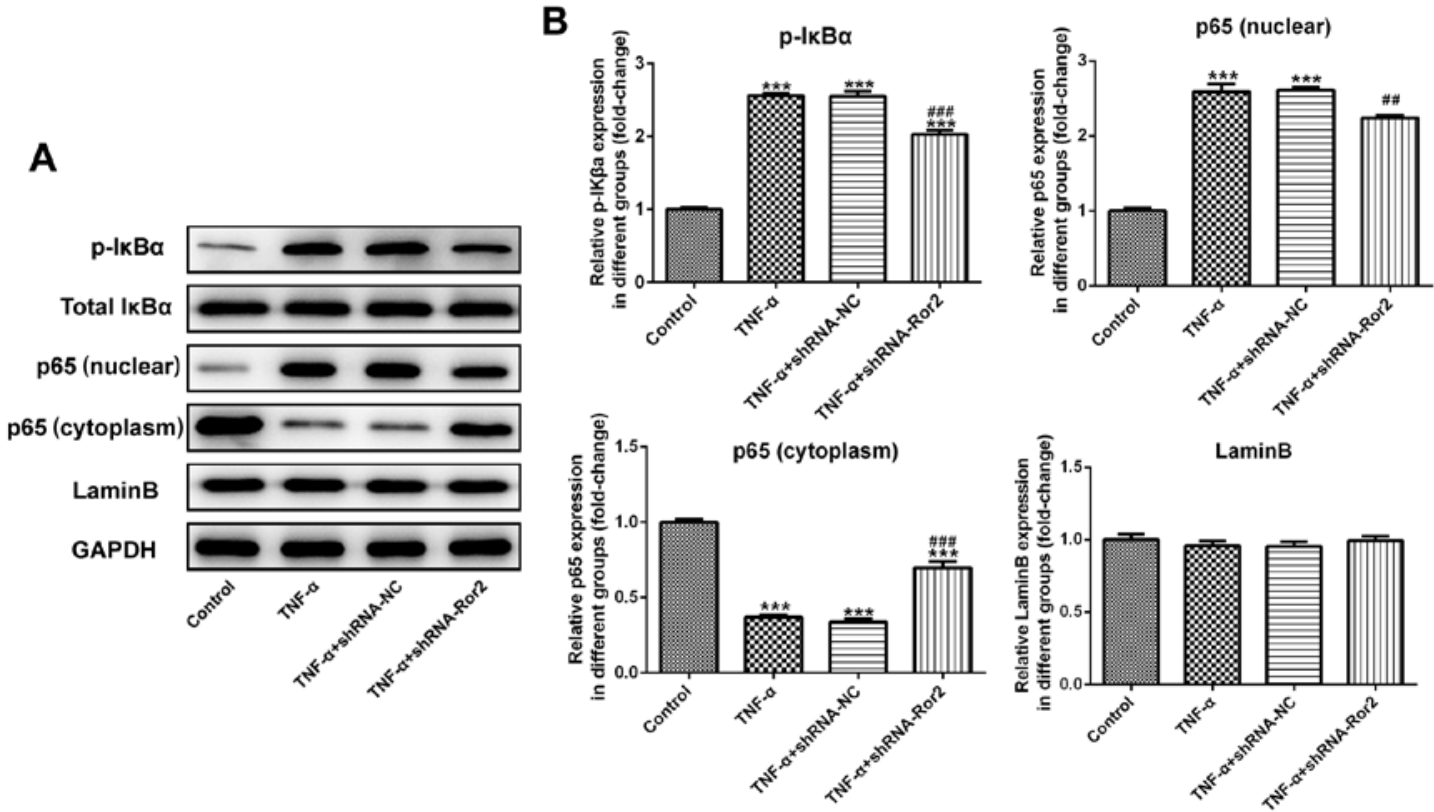

Figure 4. Ror2-knockdown inhibits the activation of NF-кB induced by TNF- $\alpha$. (A) Representative immunoblot analysis for p-IкB $\alpha$, nuclear p65, cytoplasmic p65 and lamin B in HUVECs. (B) Relative protein expression of $\mathrm{p}-\mathrm{I \kappa} \mathrm{B} \alpha$, nuclear p65, cytoplasmic p65 and lamin $\mathrm{B}$ in different groups. $\mathrm{n}=3$. ${ }^{* * *} \mathrm{P}<0.001 \mathrm{vs}$. control; ${ }^{\# \#} \mathrm{P}<0.05$ and ${ }^{\# \# \#} \mathrm{P}<0.001$ vs. TNF- $\alpha$. TNF- $\alpha$, tumor necrosis factor- $\alpha$; shRNA, short hairpin RNA; NC, negative control; p-, phosphorylated; Ror2, receptor tyrosine kinase-like orphan receptors 2; HUVEC, human umbilical vein endothelial cells.

As presented in Fig. 4, upon treatment with TNF- $\alpha$, the levels of $\mathrm{p}-\mathrm{I} \mathrm{K} \mathrm{B} \alpha$ and nuclear $\mathrm{p} 65$ significantly increased compared with controls, while p65 levels significantly decreased in the cytoplasm. However, Ror2-knockdown significantly inhibited the TNF- $\alpha$-induced increase of $\mathrm{p}-\mathrm{I \kappa} \mathrm{B} \alpha$ and recovered $\mathrm{p} 65$ expression. In the canonical NF- $\mathrm{KB}$ pathway, the release of 

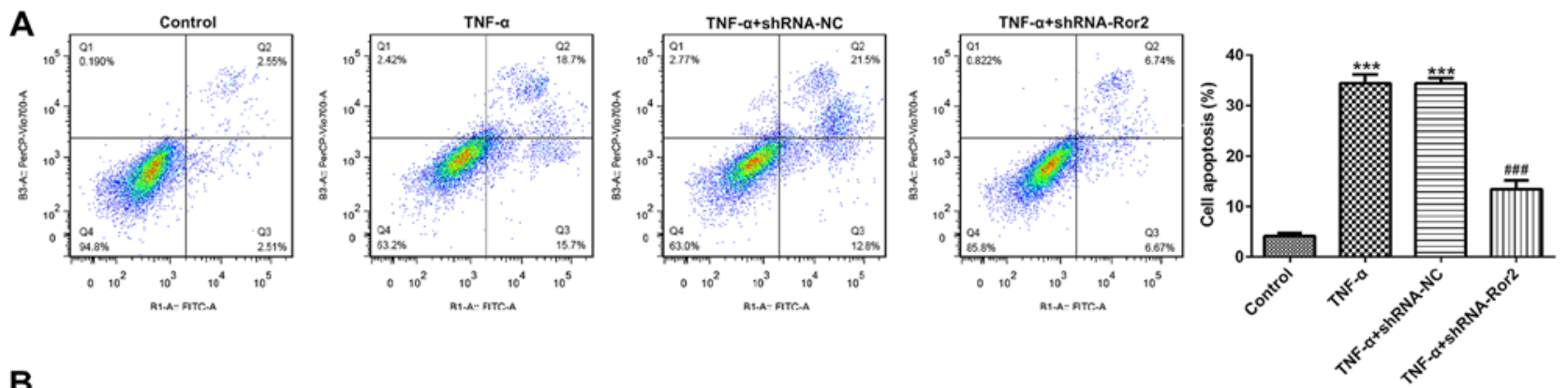

B
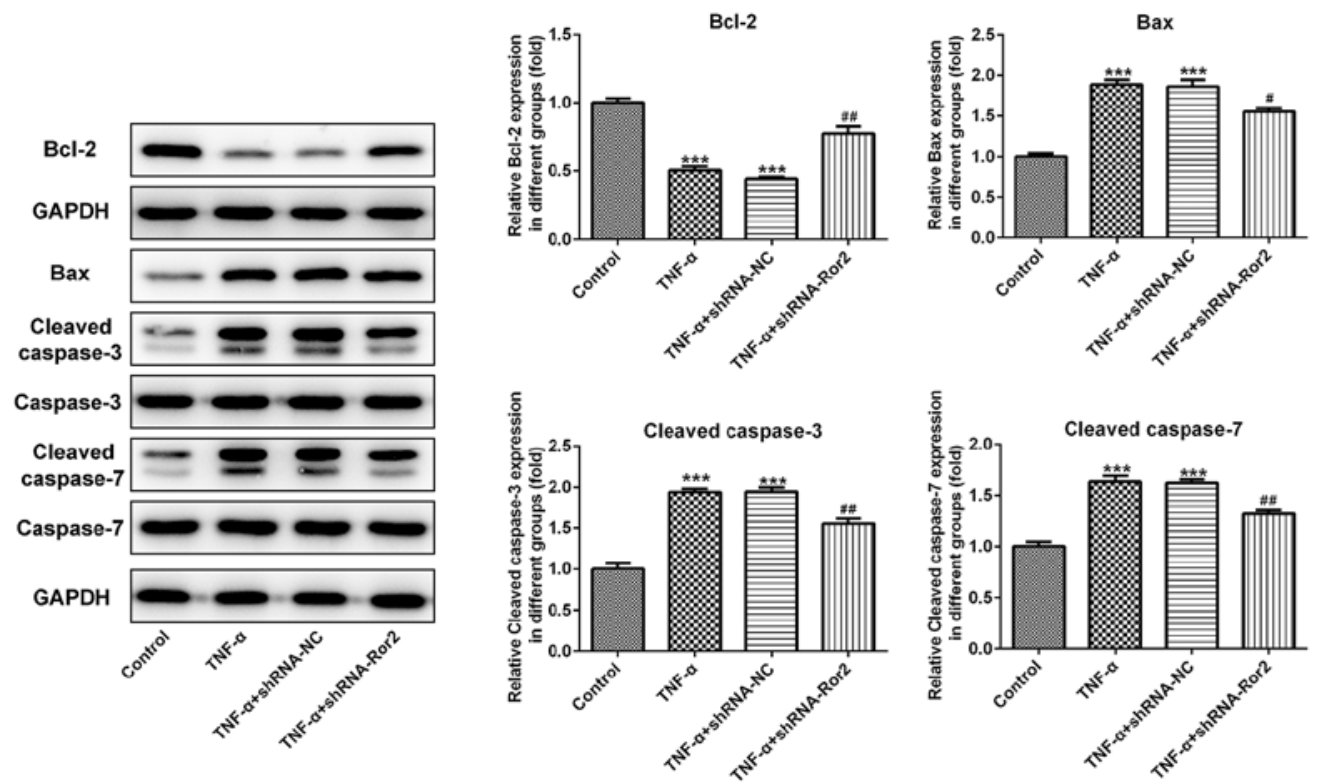

Figure 5. Ror2 knockdown reduces TNF- $\alpha$-induced HUVEC apoptosis. (A) Cell apoptosis was assessed by flow cytometry and the ratio of apoptotic cells in each group was calculated. $n=3$. (B) Representative immunoblot analysis and relative protein expression of apoptosis-related proteins in different groups. $n=3$. ${ }^{* * * *} \mathrm{P}<0.001$ vs. control; ${ }^{\#} \mathrm{P}<0.05,{ }^{\# \#} \mathrm{P}<0.01$ and ${ }^{\# \#} \mathrm{P}<0.001$ vs. TNF- $\alpha$. TNF- $\alpha$, tumor necrosis factor- $\alpha$; shRNA, short hairpin RNA; NC, negative control; Ror2, receptor tyrosine kinase-like orphan receptors 2 ; HUVEC, human umbilical vein endothelial cells.

the $\mathrm{p} 65 / \mathrm{p} 50$ complex represents the transcription activation of $\mathrm{NF}-\kappa \mathrm{B}$ pathway (29). These results revealed that knockdown of Ror 2 could prevent activation of NF- $\kappa$ B.

Knockdown of Ror2 reduces TNF- $\alpha$-induced HUVEC apoptosis. To observe the effect of Ror2-knockdown on cell apoptosis induced by TNF- $\alpha$, flow cytometry and western blotting were performed. As presented in Fig. 5, TNF- $\alpha$ resulted in a significant increase in the apoptosis rate of HUVECs compared with controls, while cells transfected with shRNA-Ror2 exhibited a lower apoptosis rate compared with the TNF- $\alpha$ group. Compared with controls, following TNF- $\alpha$ stimulation, the protein expression of Bcl-2 significantly decreased, while Bax, cleaved caspase-3 and cleaved caspase- 7 expression significantly increased, indicating the enhancement of apoptotic activity. Although caspase-7 was considered to be redundant compared with caspase 3 , studies have confirmed the crucial role of caspase 7 in apoptosis and inflammation $(30,31)$. As a downstream effector of caspase 8/9/10/1, caspase 7 needs to be evaluated during apoptosis. Furthermore, Ror2-knockdown also rescued the protein expression of these apoptosis-related proteins (Fig. 5B), suggesting the inhibitory effect of Ror2-knockdown on TNF- $\alpha$-induced HUVEC apoptosis.

Ror2 interacts with Wnt5a and can be regulated by Wnt5a. Finally, to investigate whether the protective effect of Ror2 knockdown on TNF- $\alpha$-induced vascular endothelial cell injury was mediated by its upstream ligand Wnt5a, the alteration of Wnt5a protein levels upon TNF- $\alpha$ stimulation was investigated. The results demonstrated that, consistent with previous findings $(14,32)$, Wnt5a expression was enhanced by TNF- $\alpha$ in a concentration-dependent manner (Fig. 6A), which indicated the potential modulatory effect of Wnt5a on TNF- $\alpha$-induced HUVECs. Subsequently, IP assay confirmed the interaction of Ror2 and Wnt5a in HUVECs under TNF- $\alpha$ stimulation (Fig. 6B). To further investigate the effect of Wnt5a on Ror2 expression in TNF- $\alpha$-treated HUVEC, Wnt5a was knocked down by shRNA-Wnt5a (Fig. 6C). ShRNA-Wnt5a-1 exerted a superior knockdown effect and significantly reduced the protein expression of Ror2 in HUVECs exposed to TNF- $\alpha$ compared with control and shRNA-negative control groups (Fig. 6C and D). These results suggested that Ror2 was likely to exert its functions on TNF- $\alpha$-induced HUVEC injury via binding to Wnt5a.

\section{Discussion}

Specific Wnt/receptor/co-receptor combinations are particularly important in determining downstream signaling. As a member of the receptor tyrosine kinase family, Ror2 can act as a receptor or co-receptor of Wnt5a to participate in the 
A
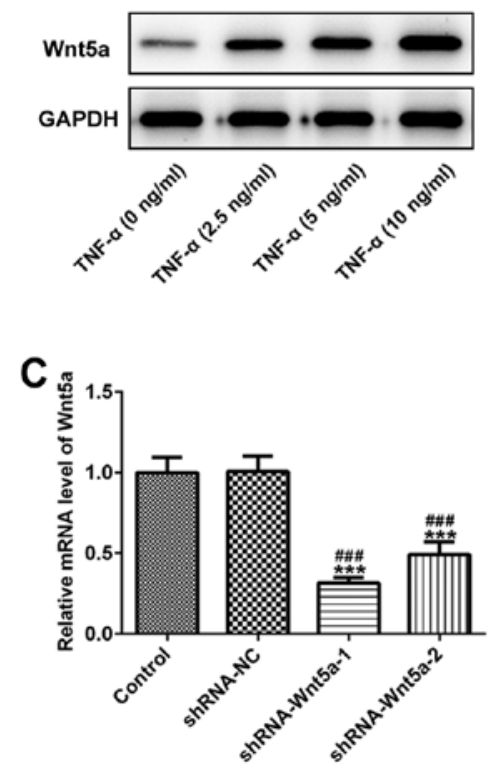

Wnt5a

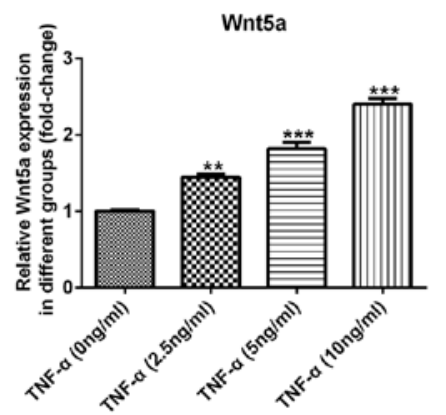

D

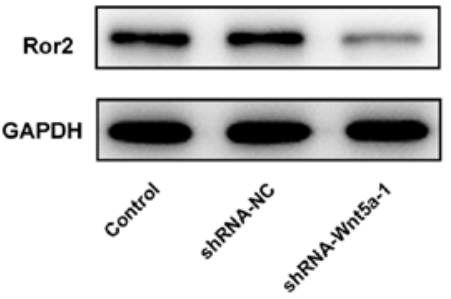

B
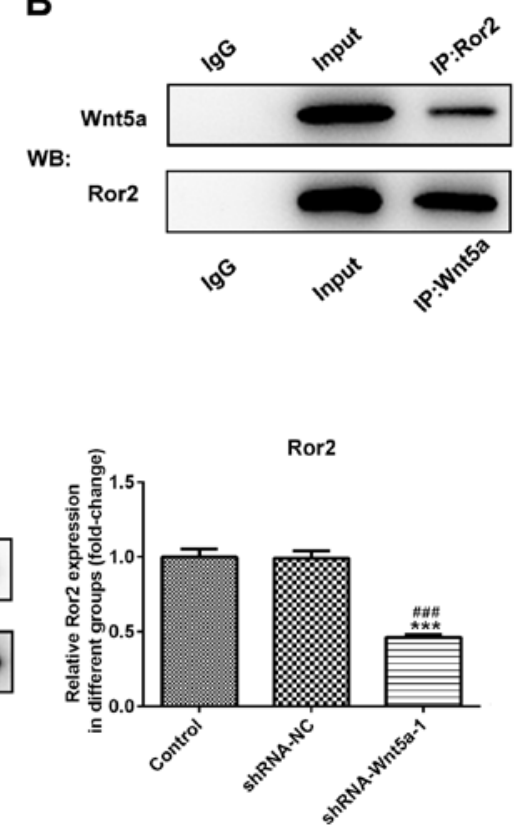

Figure 6. Interaction of Ror2 with Wnt5a. (A) Representative immunoblot analysis and relative protein expression of Wnt5a following stimulation of HUVECs with different concentrations of TNF- $\alpha . \mathrm{n}=3$. ${ }^{* *} \mathrm{P}<0.01$ and ${ }^{* * * *} \mathrm{P}<0.001 \mathrm{vs}$. TNF-a $(0 \mathrm{ng} / \mathrm{ml})$. (B) The protein interaction of Ror 2 and Wnt5a was assessed by IP. $\mathrm{n}=3$. (C) HUVECs were transfected with the indicated vectors, and the mRNA levels of Wnt5a was detected. $\mathrm{n}=3$. ${ }^{* * * *} \mathrm{P}<0.001 \mathrm{vs}$. control; ${ }^{\# \# \#} \mathrm{P}<0.001$ vs. shRNA-NC. (D) Alteration of Ror2 protein expression following Wnt5a knockdown. $n=3 .{ }^{* * *} \mathrm{P}<0.001$ vs. control; ${ }^{\# \#} \mathrm{P}<0.001$ vs. shRNA-NC. TNF- $\alpha$, tumor necrosis factor- $\alpha$; shRNA, short hairpin RNA; NC, negative control; Ror2, receptor tyrosine kinase-like orphan receptors 2; HUVEC, human umbilical vein endothelial cells; Wnt5a, Wnt family member 5a; IgG, immunoglobulin G; IP, immunoprecipitation; WB, western blot.

regulation of non-classical Wnt- $\mathrm{Ca}^{2+}$ and Wnt-PCP signaling pathways, thus mediating cell proliferation, migration, adhesion and location (33). To the best of our knowledge, the present study was the first to demonstrate the function of Ror2 in vascular endothelial cell injury and revealed that Ror2-knockdown could suppresses TNF- $\alpha$-induced inflammation and apoptosis in vascular endothelial cells.

Ror2 has been identified to be involved in the early formation of chondrocytes and may be required for cartilage and growth plate development $(34,35)$. Following more in-depth research, Ror2 was shown to be highly expressed in a variety of cancer tissues and correlated with patient prognosis (36-38). Studies have reported that Wnt5a-Ror2 signaling is involved in the regulation of inflammatory diseases, such as atherosclerosis, and Ror2 is highly expressed in atherosclerotic plaques and atherosclerotic patient sera, similar to Wnt5a $(20,27,39)$. These findings suggested that Ror2 may regulate the development of AS. Vascular endothelial cell injury is the initial step of AS and directly influences the development of AS (2). In the present study, TNF- $\alpha$ was used to induce vascular endothelial cell injury to stimulate AS in vitro. It was identified that Ror2 protein expression was upregulated by TNF- $\alpha$ in a concentration-dependent manner. Based on the present and previous findings (21-23), it has been demonstrated that Ror2 plays an important role in AS. To verify this, Ror2 was knocked down in the current study to observe whether the injury of HUVECs could be alleviated. In addition, since TNF- $\alpha$ has been demonstrated to increase Ror2 expression, the present study aimed to uncover a therapy for alleviating TNF- $\alpha$-induced injury, instead of aggravating injury, therefore, overexpression of Ror2 will not be covered in this study.
Inflammatory response is a marker of vascular endothelial cell damage (6). Upon stimulation of inflammatory factor TNF- $\alpha$, increased levels of TNF- $\alpha$, IL-1 $\beta$, IL- 6 and MCP-1 were released. Additionally, the expression levels of ICAM-1 and VCAM-1 were significantly enhanced by TNF- $\alpha$ stimulation. ICAM-1 and VCAM-1 are adhesion molecules that can bind to monocytes and lymphocytes and cause adhesion reactions (25). MCP-1 can promote the transformation of monocytes into macrophages, leading to the formation of foam cells $(40,41)$. The present results demonstrated that cells that transfected with shRNA-Ror2 exhibited lower levels of TNF- $\alpha$, IL-1 $\beta$, IL-6, MCP-1, ICAM-1 and VCAM-1 compared with normal HUVECs upon TNF- $\alpha$ stimulation, indicating the inhibitory effect on inflammation and AS progression. However, further immunofluorescence staining experiments are needed to directly reflect the enhancement of inflammation.

The NF- $\kappa \mathrm{B}$ signaling pathway can be activated by TNF- $\alpha$, thereby activating target gene transcription and generating cellular responses, such as immunity, inflammation and stress (42). The present results demonstrated that Ror2 silencing significantly inhibited the phosphorylation of IкB $\alpha$ and the nuclear translocation of p65 in TNF- $\alpha$ stimulated cells. Wnt5a has been implicated to regulate NF- $\kappa$ B signaling (42). Ror2 silencing may block the phosphorylation of $\mathrm{I} \kappa \mathrm{B} \alpha$ via preventing the transcription of downstream genes, thus affecting $\mathrm{NF}-\kappa \mathrm{B}$ signaling. In addition, knockdown of Ror2 successfully inhibited the cell apoptosis induced by TNF- $\alpha$. These results revealed that Ror2-knockdown could prevent activation of NF- $\mathrm{B}$ signaling and inhibit cell apoptosis.

The role of Wnt5a in AS has been extensively studied, however the present study aimed to investigate whether the 
effect of Ror2 on vascular endothelial cell injury could be mediated by Wnt5a. The interaction between Wnt5a and Ror2 in TNF- $\alpha$-treated HUVECs was confirmed by IP assay, and the western blotting results suggested that the protein expression of Ror2 was decreased by Wnt5a-knockdown. However, the protein level of Ror2 was not completed abolished by Wnt5a-knockdown, implying other ligands could mediate Ror2 expression, which needs to be investigated in future work. In addition, whether Wnt5a-knockdown could abolish or affect the inhibitory effect of Ror2-knockdown on vascular endothelial cell injury during the development of AS remains to be clarified. Our results confirmed that in the presence of shRNA-Wnt5a, the protein expression of Ror2 in HUVECs exposed to TNF- $\alpha$ was significantly reduced. In addition, the IP assay further confirmed their interaction. These results are sufficient to suggest that Ror2 was likely to exert its functions on TNF- $\alpha$-induced HUVEC injury via binding to Wnt5a. A recent study (27) indicated that knockdown of Wnt5a suppressed LPS-induced cholesterol accumulation and inflammatory response in VSMCs. The present study illustrated that inhibition of Wnt5a receptor-Ror 2 could also prevent TNF- $\alpha$ stimulated inflammation, NF- $\kappa \mathrm{B}$ activation and apoptosis and suggested the participation of other ligands in regulating Ror2-mediated HUVEC injury. Furthermore, AS may be driven by an innate immune response through myeloid cells. In future studies, the decline of protective $\mathrm{T}$ regulatory cells in the course of AS would be covered.

In conclusion, the present study identified Wnt5a/Ror2 as a novel regulator in TNF- $\alpha$-induced HUVECs inflammation and apoptosis. Knockdown of Ror2 may be a potential therapeutic approach in treating or relieving AS.

\section{Acknowledgements}

Not applicable.

\section{Funding}

No funding was received.

\section{Availability of data and materials}

All data generated or analyzed during this study are included in this published article.

\section{Authors' contributions}

HZ and XY conceived and designed the study. XY, SZ, HY and RS acquired the data. WG, ZG and XL analysed and interpreted the data. $\mathrm{HZ}$ and $\mathrm{XY}$ drafted the manuscript and revised it for critically important intellectual content. All authors read and approved the final manuscript.

\section{Ethics approval and consent to participate}

Not applicable.

\section{Patient consent for publication}

Not applicable.

\section{Competing interests}

The authors declare that they have no competing interests.

\section{References}

1. Schaftenaar F, Frodermann V, Kuiper J and Lutgens E: Atherosclerosis: The interplay between lipids and immune cells. Curr Opin Lipidol 27: 209-215, 2016.

2. Libby P, Ridker PM and Hansson GK: Progress and challenges in translating the biology of atherosclerosis. Nature 473: 317-325, 2011.

3. Gisterå A and Hansson GK: The immunology of atherosclerosis. Nat Rev Nephrol 13: 368-380, 2017.

4. Wolf $D$ and Ley K: Immunity and Inflammation in Atherosclerosis. Circ Res 124: 315-327, 2019.

5. Gimbrone MA Jr and García-Cardeña G: Endothelial Cell Dysfunction and the Pathobiology of Atherosclerosis. Circ Res 118: 620-636, 2016.

6. Sitia S, Tomasoni L, Atzeni F, Ambrosio G, Cordiano C, Catapano A, Tramontana S, Perticone F, Naccarato P, Camici P, et al: From endothelial dysfunction to atherosclerosis. Autoimmun Rev 9: 830-834, 2010.

7. Förstermann U, Xia N and Li H: Roles of vascular oxidative stress and nitric oxide in the pathogenesis of atherosclerosis. Circ Res 120: 713-735, 2017.

8. Eelen G, de Zeeuw P, Simons M and Carmeliet P: Endothelial cell metabolism in normal and diseased vasculature. Circ Res 116: 1231-1244, 2015.

9. Cochain $\mathrm{C}$ and Zernecke A: Macrophages in vascular inflammation and atherosclerosis. Pflugers Arch 469: 485-499, 2017.

10. Nusse $R$ and Clevers $H$ : Wnt/ $\beta$-catenin signaling, disease, and emerging therapeutic modalities. Cell 169: 985-999, 2017.

11. Matthijs Blankesteijn W and Hermans KC: Wnt signaling in atherosclerosis. Eur J Pharmacol 763 (Pt A): 122-130, 2015.

12. Krishna SM, Seto SW, Jose RJ, Li J, Morton SK, Biros E, Wang Y, Nsengiyumva V, Lindeman JH, Loots GG, et al: Wnt signaling pathway inhibitor sclerostin inhibits angiotensin II-induced aortic aneurysm and atherosclerosis. Arterioscler Thromb Vasc Biol 37: 553-566, 2017.

13. Christman MA II, Goetz DJ, Dickerson E, McCall KD, Lewis CJ, Benencia F, Silver MJ, Kohn LD and Malgor R: Wnt5a is expressed in murine and human atherosclerotic lesions. Am J Physiol Heart Circ Physiol 294: H2864-H2870, 2008.

14. Malgor R, Bhatt PM, Connolly BA, Jacoby DL, Feldmann KJ, Silver MJ, Nakazawa M, McCall KD and Goetz DJ: Wnt5a, TLR2 and TLR4 are elevated in advanced human atherosclerotic lesions. Inflamm Res 63: 277-285, 2014.

15. Bhatt PM and Malgor R: Wnt5a: A player in the pathogenesis of atherosclerosis and other inflammatory disorders. Atherosclerosis 237: 155-162, 2014.

16. Zhou XL, Zhang CJ, Peng YN, Wang Y, Xu HJ and Liu CM: ROR2 modulates neuropathic pain via phosphorylation of NMDA receptor subunit GluN2B in rats. Br J Anaesth 123: e239-e248, 2019.

17. Gordon MD and Nusse R: Wnt signaling: Multiple pathways, multiple receptors, and multiple transcription factors. J Biol Chem 281: 22429-22433, 2006.

18. Yu J, Chen L, Cui B, Widhopf GF II, Shen Z, Wu R, Zhang L, Zhang S, Briggs SP and Kipps TJ: Wnt5a induces ROR1/ROR2 heterooligomerization to enhance leukemia chemotaxis and proliferation. J Clin Invest 126: 585-598, 2016.

19. Arabzadeh S, Hossein G, Salehi-Dulabi Z and Zarnani AH: WNT5A-ROR2 is induced by inflammatory mediators and is involved in the migration of human ovarian cancer cell line SKOV-3. Cell Mol Biol Lett 21: 9, 2016.

20. Sato A, Kayama H, Shojima K, Matsumoto S, Koyama H, Minami Y, Nojima S, Morii E, Honda H, Takeda K, et al: The Wnt5a-Ror 2 axis promotes the signaling circuit between interleukin-12 and interferon- $\gamma$ in colitis. Sci Rep 5: 10536, 2015.

21. Ackers I, Szymanski C, Duckett KJ, Consitt LA, Silver MJ and Malgor R: Blocking Wnt5a signaling decreases CD36 expression and foam cell formation in atherosclerosis. Cardiovasc Pathol 34: $1-8,2018$

22. Pan W, Yu H, Huang S and Zhu P: Resveratrol Protects against TNF- $\alpha$-Induced Injury in Human Umbilical Endothelial Cells through Promoting Sirtuin-1-Induced Repression of NF-KB and p38 MAPK. PLoS One 11: e0147034, 2016. 
23. Livak KJ and Schmittgen TD: Analysis of relative gene expression data using real-time quantitative PCR and the 2(-Delta Delta C(T)) Method. Methods 25: 402-408, 2001.

24. Li Y, Sun J, Gu L and Gao X: Protective effect of CTRP6 on cerebral ischemia/reperfusion injury by attenuating inflammation, oxidative stress and apoptosis in PC12 cells. Mol Med Rep 22: 344-352, 2020.

25. Kim DH, Lee SM, Lee YJ, Yoon JJ, Tan R, Yu YC, Kang DG and Lee HS: Effect of Paeotang on tumor necrosis factor $\alpha$-induced vascular inflammation in human umbilical vein endothelial cells. Chin J Integr Med: doi.10.1007/s11655-017-2759-3.

26. Choe JY, Park KY, Lee SJ, Park SH and Kim SK: Rebamipide inhibits tumor necrosis factor- $\alpha$-induced interleukin- 8 expression by suppressing the NF- $\kappa \mathrm{B}$ signal pathway in human umbilical vein endothelial cells. Inflamm Res 59: 1019-1026, 2010.

27. Zhang CJ, Zhu N, Liu Z, Shi Z, Long J, Zu XY, Tang ZW, Hu ZY, Liao DF and Qin L: Wnt5a/Ror2 pathway contributes to the regulation of cholesterol homeostasis and inflammatory response in atherosclerosis. Biochim Biophys Acta Mol Cell Biol Lipids 1865: 158547, 2020.

28. DiDonato JA, Mercurio F and Karin M: NF- $\kappa$ B and the link between inflammation and cancer. Immunol Rev 246: 379-400, 2012.

29. Chen XJ, Zhang WN, Chen B, Xi WD, Lu Y, Huang JY, Wang YY Long J, Wu SF, Zhang YX, et al: Homoharringtonine deregulates MYC transcriptional expression by directly binding NF- $\mathrm{KB}$ repressing factor. Proc Natl Acad Sci USA 116: 2220-2225, 2019

30. Brentnall M, Rodriguez-Menocal L, De Guevara RL, Cepero E and Boise LH: Caspase-9, caspase-3 and caspase-7 have distinct roles during intrinsic apoptosis. BMC Cell Biol 14: 32, 2013.

31. Lamkanfi M and Kanneganti TD: Caspase-7: A protease involved in apoptosis and inflammation. Int J Biochem Cell Biol 42: 21-24, 2010.

32. Bhatt PM, Lewis CJ, House DL, Keller CM, Kohn LD, Silver MJ, McCall KD, Goetz DJ and Malgor R: Increased Wnt5a mRNA expression in advanced atherosclerotic lesions, and oxidized LDL treated human monocyte-derived macrophages. Open Circ Vasc J 5: 1-7, 2012.
33. Flores-Hernández E, Velázquez DM, Castañeda-Patlán MC, Fuentes-García G, Fonseca-Camarillo G, Yamamoto-Furusho JK, Romero-Avila MT, García-Sáinz JA and Robles-Flores M: Canonical and non-canonical Wnt signaling are simultaneously activated by Wnts in colon cancer cells. Cell Signal 72: 109636, 2020.

34. Takeuchi S, Takeda K, Oishi I, Nomi M, Ikeya M, Itoh K, Tamura S, Ueda T, Hatta T, Otani H, et al: Mouse Ror2 receptor tyrosine kinase is required for the heart development and limb formation. Genes Cells 5: 71-78, 2000.

35. Witte F, Chan D, Economides AN, Mundlos S and Stricker S: Receptor tyrosine kinase-like orphan receptor 2 (ROR2) and Indian hedgehog regulate digit outgrowth mediated by the phalanx-forming region. Proc Natl Acad Sci USA 107: 14211-14216, 2010.

36. Dai B, Yan $\mathrm{T}$ and Zhang A: ROR 2 receptor promotes the migration of osteosarcoma cells in response to Wnt5a. Cancer Cell Int 17: 112, 2017.

37. Henry CE, Llamosas E, Daniels B, Coopes A, Tang K and Ford CE: ROR1 and ROR2 play distinct and opposing roles in endometrial cancer. Gynecol Oncol 148: 576-584, 2018.

38. Debebe Z and Rathmell WK: Ror2 as a therapeutic target in cancer. Pharmacol Ther 150: 143-148, 2015.

39. Takahashi D, Suzuki H, Kakei Y, Yamakoshi K, Minami Y, Komori T and Nishita M: Expression of Ror2 associated with fibrosis of the submandibular gland. Cell Struct Funct 42: 159-167, 2017.

40. Murphy JM, Jeong K, Rodriguez YAR, Kim JH, Ahn EE and Lim SS:FAK and Pyk2 activity promote TNF- $\alpha$ and IL- $1 \beta$-mediated pro-inflammatory gene expression and vascular inflammation. Sci Rep 9: 7617, 2019

41. Liu JB, Jia L, Li BR, Lan LZ, Ge Q, Zhen HT and Deng HC: Adiponectin suppresses inflammatory responses at the early phase of atherosclerosis in hyperglycemic rats. Mol Med Rep 3 323-328, 2010.

42. Bergenfelz C, Medrek C, Ekström E, Jirström K, Janols H, Wullt M, Bredberg A and Leandersson K: Wnt5a induces a tolerogenic phenotype of macrophages in sepsis and breast cancer patients. J Immunol 188: 5448-5458, 2012. 